\title{
ATYPICAL PRESENTATION OF COPA MUTATION AS CAUSE OF INTERSTITIAL LUNG DISEASE IN A YOUNG INFANT
}

\author{
Carolina Quintana ${ }^{1}$, Katarzyna Saar ${ }^{2}$, Heather Tory ${ }^{2}$, and Tregony Simoneau ${ }^{3}$ \\ ${ }^{1}$ Baylor College of Medicine \\ ${ }^{2}$ University of Connecticut Health Center \\ ${ }^{3}$ Harvard Medical School
}

June 22, 2020

\begin{abstract}
A 2-month-old infant Hispanic female with failure to thrive and choking episodes was admitted with progressive worsening of respiratory effort, paroxysmal coughing, and hypoxemia. She required prolonged mechanical ventilation and post-pyloric feeding for concern of aspiration with no marked improvement of chest radiogram or CT findings. Lung biopsy performed and remarkable for lymphocytic infiltration but without a definite diagnosis. Genetic testing identified a unique heterozygous mutation in the COPA gene. She was started on rituximab and azathioprine initially and has weaned off oxygen and the ventilator. We report a unique case of interstitial lung disease secondary to COPA gene mutation without frank pulmonary hemorrhage, renal, or joint involvement upon initial presentation.
\end{abstract}

\section{INTRODUCTION}

COPA syndrome is a rare cause of interstitial lung disease (ILD) that was initially identified in 2015. It is described as a monogenic autoimmune lung and joint disorder caused by alteration of the COPA gene located in the 1q23.2 chromosome. ${ }^{1,2,3}$ This mutation results in ineffective retrograde transport from the Golgi complex to the Endoplasmic Reticulum causing increase in ER stress and ineffective autophagy ${ }^{3,4}$. Symptoms typically present prior to 5 years of age and are characterized by arthritis, renal disease, and diffuse interstitial lung disease with pulmonary hemorrhage. We present a unique case of a 2-month-old Hispanic female found to have ILD secondary to COPA gene mutation without frank pulmonary hemorrhage or joint involvement.

\section{CASE PRESENTATION}

A former full-term female presented with failure to thrive, cough, and hypoxemia (Sp02 70-80\%) that progressed to acute respiratory failure requiring endotracheal intubation and mechanical ventilation. Repeat chest radiographs were notable for right upper lobe, lingular, and left lower lobe opacities that did not respond to multiple antibiotic courses. Initial flexible bronchoscopy was notable for significant tracheobronchomalacia with at least $50 \%$ collapse of distal trachea and proximal right mainstem bronchus. Additional work up included a normal brain MRI and initial echocardiogram. Chest CT was notable for multiple ground glass opacities suggestive of ILD or chronic aspiration (Figure 1). Given concern for aspiration she was advanced to post pyloric feeding. Lung biopsy was done and notable for bronchiolar lymphoid hyperplasia, chronic bronchiolitis, mild alveolar simplification, lymphocytic infiltration and interspersed plasma cells, architectural remodeling, and distortion of small airways (Figure 2). Electron microscopy noted normal lamellar body ultrastructure. Due to concern for ILD and lack of clinical improvement with post pyloric 
feeds, she was started on methylprednisolone pulses $(10 \mathrm{mg} / \mathrm{kg}$ for 3 days, monthly) for 6 months. Absolute CD3, CD3/CD4, CD3/CD8, and NK cells were low. She remained admitted until 9 months of age and was discharged with gastrojejunal feeds, tracheostomy, oxygen supplementation, and ventilator support. Her hospital course was complicated by secondary hypertension and left ventricular hypertrophy that resolved with steroid wean. Genetic studies sent prior to discharge resulted in heterozygous pathogenic variant in COPA gene (E241K ${ }^{5}$ (NM_001098398: exon 9: c.G721A)) reported to be extremely rare and not present in 60,000 unrelated individuals in the ExAC database (http://exac.broadinstitute.org/). Follow up chest $\mathrm{CT}$ at 15 months of age demonstrated progression of findings with patchy areas of consolidation and air bronchograms. Traction bronchiectasis were also seen peripherally in the lower lobes (Figure 1). Repeat bronchoscopy noted improvement of bronchomalacia. Around 22 months of age, she was noted to be ANA positive (1:320) and p-ANCA positive (1:160). Rheumatoid factor negative. No evidence of vasculitis or arthritis at time of evaluation. She was started on rituximab and azathioprine as early treatment to prevent progression of pulmonary hemorrhage, as well as renal and joint disease ${ }^{6}$. Due to adverse effects, immunosuppressive therapy was changed to methotrexate weekly. At 3 years of age, chest CT exhibited scattered nodules, diffuse bronchial thickening, and air trapping. Repeat bronchoscopy and BAL showed blood tinged secretions with $>100$ hemosiderin laden macrophages. Routine surveillance labs indicated increased ANA to 1:1280 and ANCA >1:640. Given these findings, the methotrexate dose was increased. Additional immunosuppressive therapy is currently being considered. Meanwhile, she has clinically improved and has weaned off oxygen and no longer required ventilator support. She has digital clubbing on exam but has excellent growth and development.

\section{DISCUSSION}

COPA syndrome is a rare mutation of the coatomer associated protein subunit alpha gene $(\mathrm{COP} \alpha)$ causing adaptive immune dysregulation. The cases in the literature describe ILD, pulmonary hemorrhage, arthritis, and nephropathy. This autosomal dominant entity is likely presenting as a de novo mutation in this young child as no other family members are affected. The initial lack of pulmonary hemorrhage and arthritis, as well as her age represent a novel presentation of the disease and speak to the important contribution genetic testing has made in the diagnosis of ILD. Current literature describes molecular mechanisms of the syndrome however due to twenty-one case reports described, ${ }^{5,7,8}$ clinical progression and surveillance methods are not yet described. Inflammatory markers such as ESR, C-reactive protein, complete blood count, ANA, ANCA, along with imaging and bronchoscopy with lavage cell counts have been useful markers of disease activity in this patient. However additional research is needed to better define disease markers, treatment options, and long-term prognosis.

\section{Conclusions}

Although the identification of a COPA gene mutation in this patient likely explains her underlying ILD, her presentation remains significantly different from those previously described. Therefore, this case will provide a significant contribution to the current knowledge of this disease and it emphasizes the lack of knowledge in surveillance methodologies which need to be studied.

\section{References}

1. Tsui JL, Estrada OA, Deng Z, et al. Analysis of pulmonary features and treatment approaches in the COPA syndrome. ERJ Open Res . 2018;4(2):00017-02018. doi:10.1183/23120541.00017-2018

2. Almeida de Jesus A, Goldbach-Mansky R. Newly recognized mendelian disorders. Curr Opin Rheumatol . 2015;27:511-519. doi:10.1097/BOR.0000000000000207

3. Vece TJ, Watkin LB, Nicholas SK, et al. Copa Syndrome: a Novel Autosomal Dominant Immune Dysregulatory Disease. J Clin Immunol . 2016;36(4):377-387. doi:10.1007/s10875-016-0271-8

4. Watkin LB, Jessen B, Wiszniewski W, et al. COPA mutations impair ER-Golgi transport and cause hereditary autoimmune-mediated lung disease and arthritis. Nat Genet . 2015. doi:10.1038/ng.3279 
5. COATOMER PROTEIN COMPLEX, SUBUNIT ALPHA; COPA. 601924. Online Mendelian Inheritance in Man, OMIM@. https://omim.org/. Published 2015.

6. Volpi S, Tsui J, Mariani M, et al. Type I interferon pathway activation in COPA syndrome. Clin Immunol . 2018;187:33-36. doi:10.1016/j.clim.2017.10.001

7. Noorelahi R, Perez G, Otero HJ. Imaging findings of Copa syndrome in a 12-year-old boy. Pediatr Radiol . 2018;48(2):279-282. doi:10.1007/s00247-017-3961-3

8. Jensson BO, Hansdottir S, Arnadottir GA, et al. COPA syndrome in an Icelandic family caused by a recurrent missense mutation in COPA.BMC Med Genet . 2017. doi:10.1186/s12881-017-0490-8

9. Prenzel F, Harfst J, Schwerk N, et al. Lymphocytic interstitial pneumonia and follicular bronchiolitis in children: A registry-based case series. Pediatr Pulmonol . 2020;55(4):909-917. doi:10.1002/ppul.24680
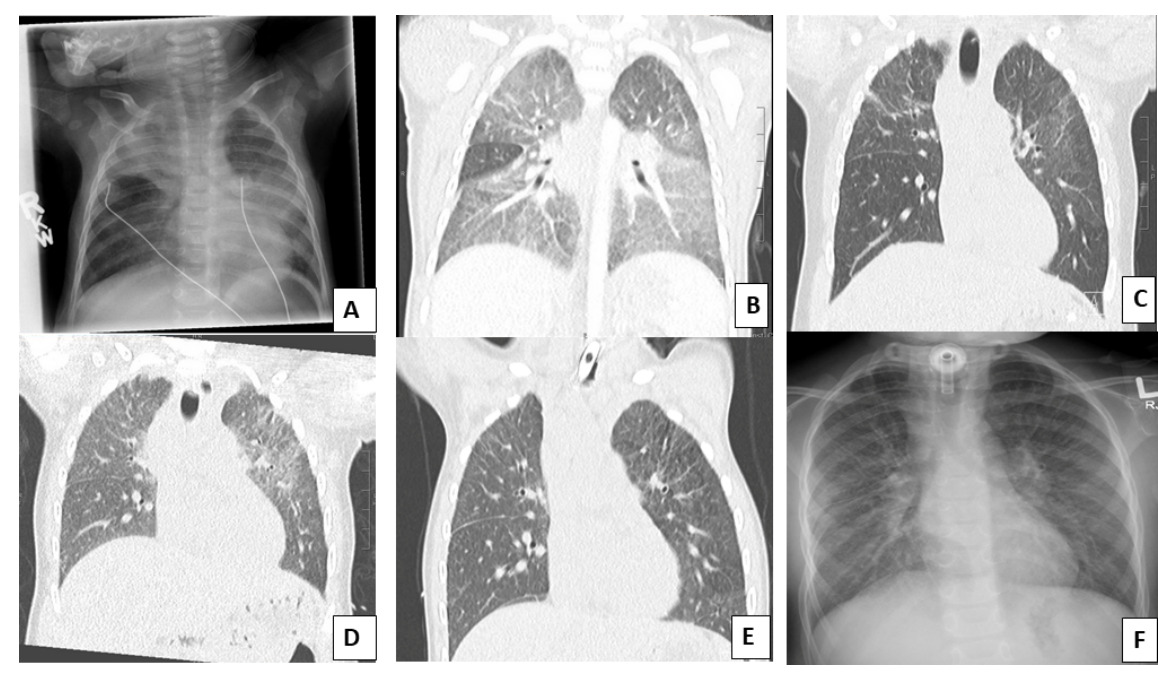
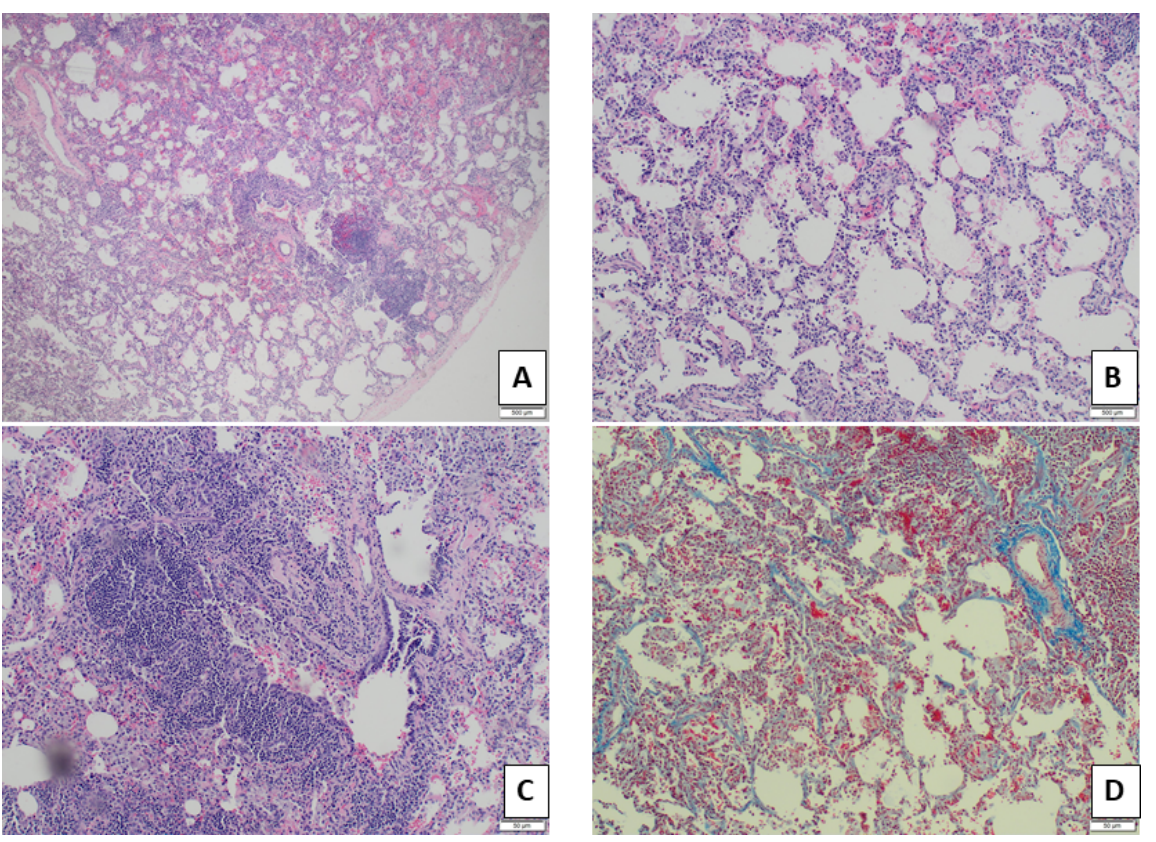